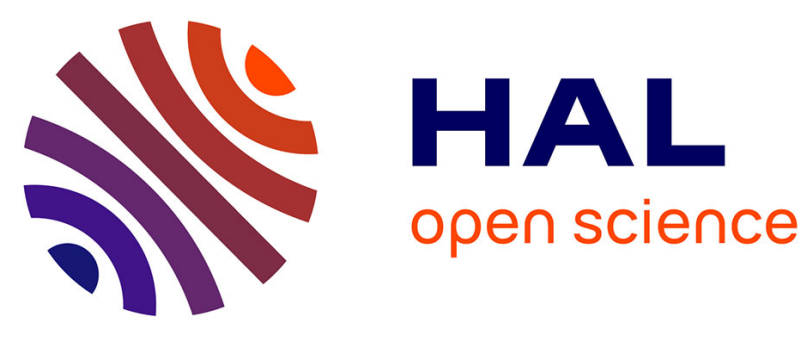

\title{
SAR Images Refocusing and Scattering Center Detection for Infrastructure Monitoring
}

\author{
Andrei Anghel, Gabriel Vasile, Cornel Ioana, Remus Cacoveanu, Silviu \\ Ciochina, Jean-Philippe Ovarlez, Rémy Boudon, Guy d'Urso
}

\section{To cite this version:}

Andrei Anghel, Gabriel Vasile, Cornel Ioana, Remus Cacoveanu, Silviu Ciochina, et al.. SAR Images Refocusing and Scattering Center Detection for Infrastructure Monitoring. RadarConf14 - 2014 IEEE Radar Conference, May 2014, Cincinnati, United States. pp.334-339, 10.1109/RADAR.2014.6875610 . hal-01065821

\section{HAL Id: hal-01065821 \\ https://hal.science/hal-01065821}

Submitted on 18 Sep 2014

HAL is a multi-disciplinary open access archive for the deposit and dissemination of scientific research documents, whether they are published or not. The documents may come from teaching and research institutions in France or abroad, or from public or private research centers.
L'archive ouverte pluridisciplinaire HAL, est destinée au dépôt et à la diffusion de documents scientifiques de niveau recherche, publiés ou non, émanant des établissements d'enseignement et de recherche français ou étrangers, des laboratoires publics ou privés. 


\title{
SAR Images Refocusing and Scattering Center Detection for Infrastructure Monitoring
}

\author{
Andrei Anghel ${ }^{* \dagger}$, Gabriel Vasile ${ }^{\dagger}$, Cornel Ioana ${ }^{\dagger}$, Remus Cacoveanu* $^{*}$, Silviu Ciochină ${ }^{*}$, Jean-Philippe Ovarlez ${ }^{\ddagger}$, \\ Rémy Boudon ${ }^{\S}$, Guy d'Urso ${ }^{\S}$ \\ *University POLITEHNICA of Bucharest, 061071 Bucharest, ROMANIA, Email: andrei.anghel@munde.pub.ro \\ ${ }^{\dagger}$ Grenoble-Image-sPeach-Signal-Automatics Lab, CNRS / Grenoble INP, 38402 Grenoble Cedex, FRANCE \\ ‡DEMR/TSI, ONERA, 91761 Palaiseau, FRANCE \\ §Électricité de France, EDF DTG / R\&D, FRANCE
}

\begin{abstract}
Infrastructure monitoring applications can require the tracking of slowly moving points of a certain structure. Given a certain point from a structure to be monitored, in the context of available SAR products where the image is already focused in a slant range - azimuth grid, it is not obvious if this point is the scattering center, if it is in layover or if it is visible from the respective orbit. This paper proposes a refocusing procedure of SAR images on a set of measured points among with a 4D tomography based scattering center detection. The refocusing procedure consists of an azimuth de-focusing followed by a modified back-projection on the given set of points. The presence of a scattering center at the given positions is detected by computing the local elevation-velocity plane for each point and testing if the main response is at zero elevation. The refocusing and scattering center detection algorithm is validated on real data acquired with the TerraSAR-X satellite during March-June 2012. The mean displacement velocities of the detected scatterers show good agreement with the in-situ measurements.
\end{abstract}

\section{INTRODUCTION}

In infrastructure monitoring, it can be often necessary to track the slow displacements of certain points of a given structure (building, water dam, landslide, etc.). This can be done using current spaceborne civil sensors, such as TerraSAR-X and TanDEM-X provided their short wavelength of $3.1 \mathrm{~cm}$, the short revisit time of 11 days and especially the $1 \mathrm{~m}$ resolution in spotlight mode [1], [2]. Still, given a certain infrastructure element, from one acquisition geometry only one side of the structure can be observed. Due to typical side-look effects (layover ambiguities, multi-path scattering effects or shadowing) it is not always clear from what points does the main response return, where is their scattering center positioned, which of them are visible from the respective orbit and consequently which of them can be accurately monitored. If the coordinates of a number of points from the structure are known with at least centimeter accuracy (measured with GPS or LIDAR techniques) their response (if there is any) could be determined if the raw data were focused on a 3-dimensional grid containing precisely this points. However, in most cases in the delivered products the SAR images are already focused on a slant range-azimuth grid which is not related to any specific scatterer.

Since the availability and processing of spaceborne raw SAR data is not very convenient, this paper proposes a method for refocusing the SAR data on a given grid containing the points of a structure that needs to be monitored. Moreover, a techinque to identify the real scatterers from the given grid (which provide the main response and are not faded by layover) in a stack of refocused images is developed in the context of differential tomography [3], [4], [5]. This is obtained by exploiting the fact that each refocused scattering center will be at zero elevation in the local elevation-velocity (EV) plane.

To achieve an accuracy of the grid focusing in the centimeter range a set of corrections are necessary regarding the atmospheric delays (for X-band the tropospheric delay can introduce a range error of around $3 \mathrm{~m}$, while the ionospheric delay is non significant) and different offsets of the sensors (as described in [6] for TerraSAR-X products). An advantage of the refocusing approach on a specific grid is that no shifting or resampling (as part of the coregistration process) are needed because the samples get automatically aligned by refocusing each image on the same grid and the interferometric phase can be obtained as the phase difference of corresponding points. Furthermore, in comparison with the classical coregistered inteferograms, a highly reflective scatterer with known coordinates that needs to be tracked (a mounted corner reflector for instance) cannot have an inconvenient off-grid position for processing in the SAR image (e.g.: to be at the edge of 4 neighboring pixels), it will always be placed in the center of the resolution cell on the new grid and its relative displacements will be accurately measured. The off-grid reflectors problem was also addressed in [7] where an algorithm for finding the reflectivity center is proposed. However, in the case of refocusing on a given grid, the purpose is to test if the scattering center is at the specified position (known with centimeter accuracy).

The refocusing technique is investigated with a set of images acquired by the TerraSAR-X satellite over the Puylaurent dam in France between March-June 2012.

The remaining of the paper is organized as follows. Section II presents the refocusing algorithm and is divided in two parts. The azimuth defocusing is discussed first. Then, the refocusing on a given grid and the scattering center detection is described. Section III shows the results of the refocusing procedure 
applied to TerraSAR-X data. Finally, the conclusions are stated in Section IV.

\section{REFOCUSING ALGORITHM AND SCATTERING CENTER DETECTION}

The refocusing algorithm consists of two steps: a defocusing in azimuth of the initial SAR image and a modified backprojection algorithm which focuses the data on the given grid. The block diagram of the procedure is shown in Fig. 1 and all the operations are described according to the flow on the processing chain. After the image is refocused, the scattering centers are detected using the differential tomography framework.

\section{A. AZIMUTH DEFOCUSING}

The defocusing procedure is actually based on a reversed version of the Spectral Analysis (SPECAN) processing used for azimuth focusing [8], [9]. This approach is possible because the azimuth scaling consists only of Fourier transforms and complex multiplications which are reversible. The first operation is to select from the initial image the slant rangeazimuth region containing the target. Because the range compression is not modified during the processing, the selected region can be cropped in range in order to reduce the computation time.

In the case of spotlight SAR images the azimuth sampling frequency is larger then the raw data pulse repetition frequency (PRF) in order to cover the complete spotlight bandwidth. In the defocusing procedure the sampling frequency is the one of the SAR image, so the subaperture approach used for realtime focusing [10] doesn't need to be employed. Therefore the subaperture recombination from the focusing algorithm in [9] can be skipped (it was necessary only to keep the PRF at the value from the raw data acquisition just until the final azimuth Fourier transform). An advantage of processing the entire aperture is that the Doppler centroid will have a very small value which minimizes the azimuth time shifts caused by the azimuth scaling.

The focused bidimensional signal for $\mathrm{N}$ scatterers (equal to the number of pixels of the cropped SAR image) can be written as:

$$
\begin{aligned}
s_{f}(t, \tau)= & \sum_{i=1}^{N} A_{i} \exp \left(-j 2 \pi f_{c} \tau\right) \operatorname{sinc}\left[\pi B_{r}\left(\tau-\tau_{i}\right)\right] \\
& \times \operatorname{sinc}\left[\pi B_{a z}\left(t-t_{i}\right)\right],
\end{aligned}
$$

where $t$ is the azimuth (slow) time axis, $\tau$ is the slant range (fast) time axis, $B_{a z}$ is the total azimuth bandwidth, $B_{r}$ is the range chirp bandwidth, $f_{c}$ is the central frequency and $A_{i}, t_{i}, \tau_{i}$ represent respectively the complex amplitude, the zero Doppler azimuth time and the slant range delay at closest approach for the scatterer $i$. For simplicity, rectangular windows for both range and azimuth data are assumed, hence the sinc functions in (1). In the SPECAN processing each zero Doppler azimuth time from the focused image is actually linked to an azimuth frequency $f_{a}$ by the scaling Doppler rate $k_{a, s c l}$ :

$$
t=-\frac{f_{a}}{k_{a, s c l}}
$$

and the image can be regarded as a function of azimuth frequency and fast time:

$$
\begin{aligned}
& s\left(f_{a}, \tau\right)=s_{f}\left(-\frac{f_{a}}{k_{a, s c l}}, \tau\right)=\sum_{i=1}^{N} A_{i} \exp \left(-j 2 \pi f_{c} \tau\right) \\
& \times \operatorname{sinc}\left[\pi B_{r}\left(\tau-\tau_{i}\right)\right] \operatorname{sinc}\left[\pi \frac{B_{a z}}{k_{a, s c l}}\left(f_{a}+k_{a, s c l} t_{i}\right)\right] .
\end{aligned}
$$

The first multiplication in the processing is made for phase preserving given the subsequent processing steps and is described by a quadratic phase term:

$$
H_{q u}\left(f_{a}\right)=\exp \left(j \pi \frac{f_{a}{ }^{2}}{k_{a, s c l}}\right) .
$$

Next an azimuth inverse Fourier transform is applied and leads to a bi-dimensional signal in the azimuth-fast time domain (computed analytically in a similar fashion as in [11] for the residual video phase removal):

$$
\begin{aligned}
& s_{1}(t, \tau)=C_{1} \sum_{i=1}^{N} A_{i} \exp \left(-j 2 \pi f_{c} \tau\right) \operatorname{sinc}\left[\pi B_{r}\left(\tau-\tau_{i}\right)\right] \\
& \quad \times r e c t\left[\frac{k_{a, s c l}}{B_{a z}}\left(t-t_{i}\right)\right] \exp \left(j \pi k_{a, s c l}\left(-2 t_{i} t+t_{i}{ }^{2}\right)\right) .
\end{aligned}
$$

By chirping $s_{1}(t, \tau)$ with the function

$$
H_{c h i r p}=\exp \left(j \pi k_{a, s c l} t^{2}\right)
$$

the resulting signal becomes a sum of chirp functions in azimuth having the zero frequency points at the closest approach azimuth times of each target:

$$
\begin{aligned}
& s_{1, \text { chirp }}(t, \tau)=C_{1} \sum_{i=1}^{N} A_{i} \exp \left(-j 2 \pi f_{c} \tau\right) \operatorname{sinc}\left[\pi B_{r}\left(\tau-\tau_{i}\right)\right] \\
& \times \operatorname{rect}\left[\frac{k_{a, s c l}}{B_{a z}}\left(t-t_{i}\right)\right] \exp \left[j \pi k_{a, s c l}\left(t-t_{i}\right)^{2}\right] .
\end{aligned}
$$

Given the support of the rectangular window in (7) and that for a SAR image with azimuth extent $t_{\text {scene }}$ the closest approach azimuth times can vary between $-t_{\text {scene }} / 2$ and $t_{\text {scene }} / 2$ the azimuth support should respect the condition

$$
T_{a z} \geq t_{\text {scene }}+\frac{B_{a z}}{\left|k_{a, s c l}\right|} .
$$

Taking into account (2) and that the azimuth sampling frequency $F_{s}$ is fixed (the equivalent PRF) the scaling Doppler rate should be chosen such that

$$
\left|k_{a, s c l}\right| \leq \frac{F_{s}-B_{a z}}{t_{\text {scene }}}
$$

and the corresponding number of azimuth points (obtained by zero padding the initial data) is

$$
N_{a z}=\left[\frac{F_{s}^{2}}{\left|k_{a, s c l}\right|}\right] \text {. }
$$




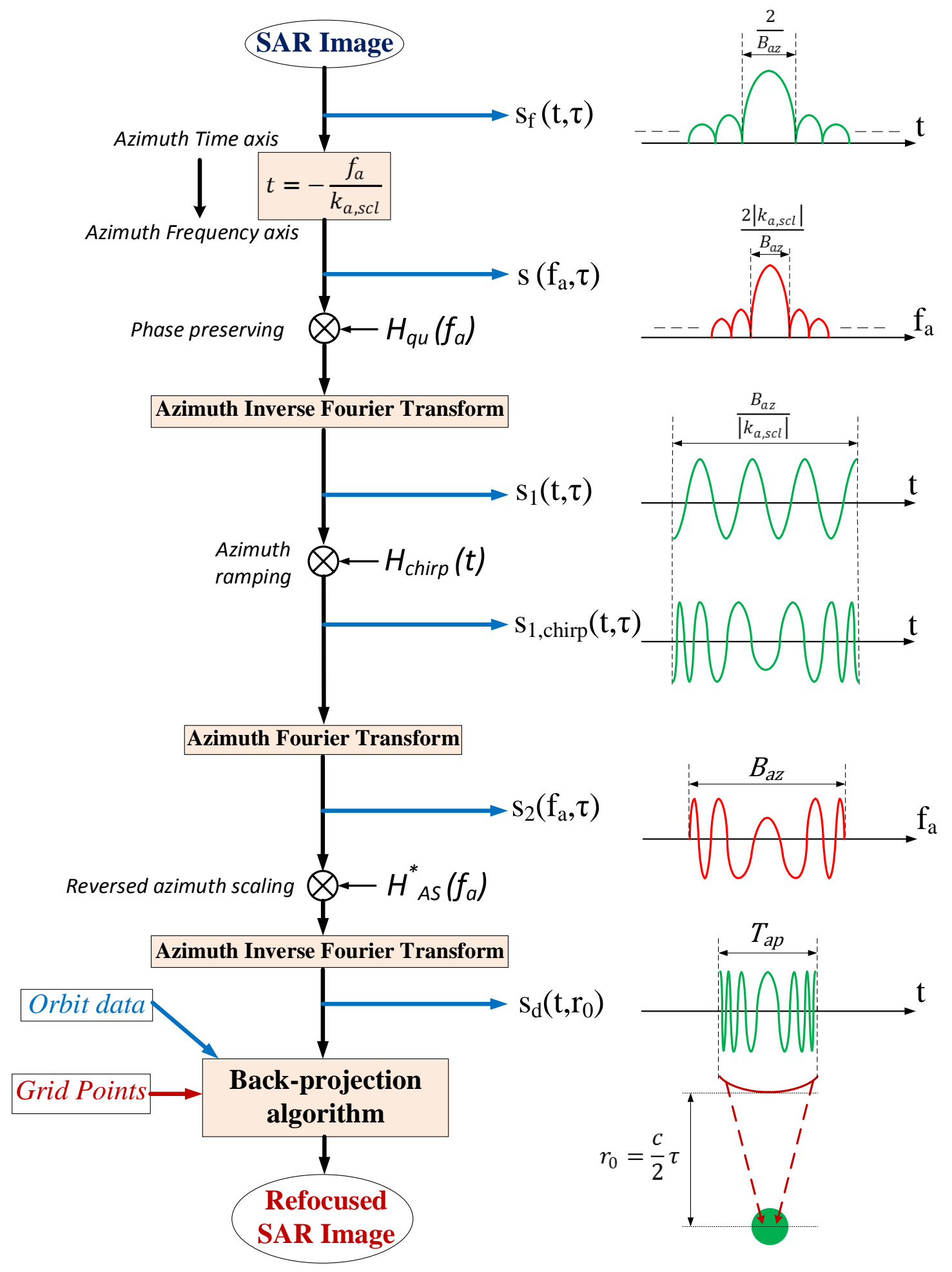

Fig. 1. Block diagram of the refocusing algorithm and qualitative representation of the real part of certain intermediate signals in the case of a single scatterer. 
Because $N_{a z}$ has to be an integer (and eventually a power of 2 ) the scaling Doppler rate can be recomputed after the proper number of points is chosen.

In the next step an azimuth Fourier transform is applied to the signal in (7) using the principle of stationary phase (PSP) [11], [12]. The PSP can be applied because the signal is a sum of chirp functions each of which having one stationary point of the phase at the zero-frequency azimuth time $t_{i}$. Moreover, each stationary point is non-degenerate because $k_{a, s c l}$ is nonzero. The result is written as:

$$
\begin{aligned}
& s_{2}\left(f_{a}, \tau\right)=C_{2} \sum_{i=1}^{N} A_{i} \exp \left(-j 2 \pi f_{c} \tau\right) \operatorname{sinc}\left[\pi B_{r}\left(\tau-\tau_{i}\right)\right] \\
& \times \operatorname{rect}\left[\frac{f_{a}}{B_{a z}}\right] \exp \left(-j \pi \frac{f_{a}{ }^{2}}{k_{a, s c l}}\right) \exp \left(-j 2 \pi f_{a} t_{i}\right) .
\end{aligned}
$$

The signal in (11) is multiplied with a complex conjugated version of the azimuth scaling function [9] expressed as

$$
\begin{aligned}
H_{A S}^{*}\left(f_{a}, \tau\right) & =\exp \left(j \pi \frac{f_{a}{ }^{2}}{k_{a, s c l}}\right) \\
& \times \exp \left[-j 2 \pi f_{c} \tau\left(\sqrt{1-\left(\frac{f_{a} \lambda}{2 v_{0}}\right)^{2}}-1\right)\right],
\end{aligned}
$$

where $\lambda$ is the wavelength at central frequency and $v_{0}$ is the zero Doppler azimuth velocity. This operation converts the chirp's quadratic phase to the original range dependent (through the fast time $\tau$ ) hyperbolic phase history. The signal takes the form:

$$
\begin{aligned}
& s_{2, h y p}\left(f_{a}, \tau\right)=C_{2} \sum_{i=1}^{N} A_{i} \exp \left(-j 2 \pi f_{c} \tau\right) \operatorname{sinc}\left[\pi B_{r}\left(\tau-\tau_{i}\right)\right] \\
& \times \operatorname{rect}\left[\frac{f_{a}}{B_{a z}}\right] \exp \left[-j 2 \pi f_{c} \tau\left(\sqrt{1-\left(\frac{f_{a} \lambda}{2 v_{0}}\right)^{2}}-1\right)\right] \\
& \times \exp \left(-j 2 \pi f_{a} t_{i}\right) .
\end{aligned}
$$

The last step is an azimuth inverse Fourier transform computed for each term of the sum in (13) using the Fourier transform pair in [13]. After this transform the azimuth de-focused bidimensional signal has the form:

$$
\begin{aligned}
& s_{d}\left(t, r_{0}\right)=C \sum_{i=1}^{N} A_{i} \operatorname{sinc}\left[\frac{2 \pi B_{r}}{c}\left(r_{0}-r_{0, i}\right)\right] \\
& \times \operatorname{rect}\left[\frac{t-t_{i}}{T_{a p}}\right] \exp \left(-j \frac{4 \pi}{\lambda} \sqrt{r_{0}^{2}+\left[v_{0}\left(t-t_{i}\right)\right]^{2}}\right),
\end{aligned}
$$

where $c$ is the speed of light and $T_{a p}$ is the equivalent synthetic aperture (illumination) time [1]. In (14) each fast time $\tau$ was written as $2 r_{0} / c$, where $r_{0}$ is the corresponding closest approach distance.

\section{B. GRID FOCUSING AND SCATTERING CENTER DETEC- TION}

Notice that the signal in (14) has the natural hyperbolic phase history for each target and is not affected by range migration. The discrete azimuth de-focused signal can be written as $s_{d}[m, n]=s_{d}(m \delta t, n \delta r)$, where $\delta t=\frac{1}{F_{s}}$ is the azimuth time spacing and $\delta r$ the range spacing. Seen as a matrix, $s_{d}[m, n]$ has on each column the phase history for a certain closest approach slant range and each line contains a range profile.

The grid focusing procedure starts by extracting the annotated orbit data [14]. The envisaged geometry is shown in Fig. 2(a). The unit vector $\overrightarrow{\boldsymbol{u}}$ of the azimuth direction is computed as the normalized velocity vector of the satellite at the azimuth time of the image center. For an azimuth resolution larger than $1 \mathrm{~m}$ the straight line trajectory approximation is satisfactory (the curved orbit correction is needed only for staring spotlight mode [15]). The position of the satellite's antenna phase center (APC) at a given azimuth time $t$ can be written as:

$$
\overrightarrow{\boldsymbol{r}}_{\boldsymbol{a}}(t)=\overrightarrow{\boldsymbol{r}}_{\boldsymbol{a}, \mathbf{0}}+v_{0} t \overrightarrow{\boldsymbol{u}}
$$

where $\overrightarrow{\boldsymbol{r}}_{\boldsymbol{a}, \mathbf{0}}$ is the APC position vector at $t=0$. For a given target having the position vector $\overrightarrow{\boldsymbol{r}}_{\boldsymbol{k}}$, the closest approach distance to the synthetic aperture is given by

$$
r_{0, k}=\left\|\left(\overrightarrow{\boldsymbol{r}}_{\boldsymbol{a}, \mathbf{0}}-\overrightarrow{\boldsymbol{r}}_{\boldsymbol{k}}\right)-\left[\left(\overrightarrow{\boldsymbol{r}}_{\boldsymbol{a}, \mathbf{0}}-\overrightarrow{\boldsymbol{r}}_{\boldsymbol{k}}\right) \cdot \overrightarrow{\boldsymbol{u}}\right] \overrightarrow{\boldsymbol{u}}\right\|
$$

The response of this target is computed using a time-domain back-projection algorithm (matched filtering based technique) [16], [17], [18], [19] modified for data with no range cell migration having the phase history of a point target on a single column of the matrix $s_{d}[m, n]$. Hence, the target response is computed as

$$
g\left(\overrightarrow{\boldsymbol{r}}_{\boldsymbol{k}}\right)=\sum_{m=M_{-}}^{M_{+}} s_{d}\left(m \delta t, r_{0, k}\right) \exp \left(j \frac{4 \pi f_{c}}{c}\left\|\overrightarrow{\boldsymbol{r}}_{\boldsymbol{a}}(m \delta t)-\overrightarrow{\boldsymbol{r}}_{\boldsymbol{k}}\right\|\right),
$$

where $M_{-}$and $M_{+}$are the limits corresponding to the total illumination duration of the respective scene (composed from the scene duration $t_{\text {scene }}$ and the equivalent synthetic aperture time $\left.T_{a p}\right)$. If the scene center has the azimuth time $t_{0}$, the summation limits are

$$
M_{ \pm}=\left[F_{s}\left(t_{0} \pm \frac{t_{\text {scene }}}{2} \pm \frac{T_{a p}}{2}\right)\right] .
$$

Notice that $r_{0, k}$ in (17) is not necessarily on the range grid (is not written as $n \delta r)$ and hence the value of $s_{d}\left(m \delta t, r_{0, k}\right)$ is interpolated in the algorithm from the matrix $s_{d}[m, n]$. The refocusing is implemented by applying (17) to each point from the given set.

For two refocused images, the stable scatterers could be detected by classical coherence evaluation on a vicinity of each refocused point. However, in order to determine if the reflecting scattering center is actually at the given point an approach based on a series of acquisitions is needed in order to compute an elevation aperture. Considering the 4D SAR imaging model in [3] for each scatterer from the given grid 


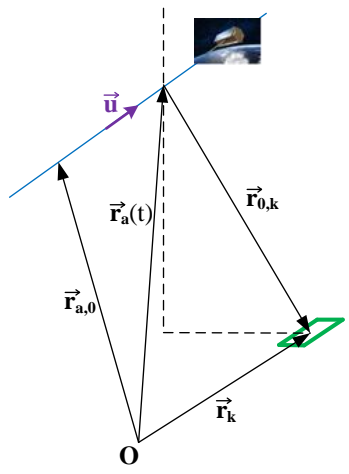

(a)

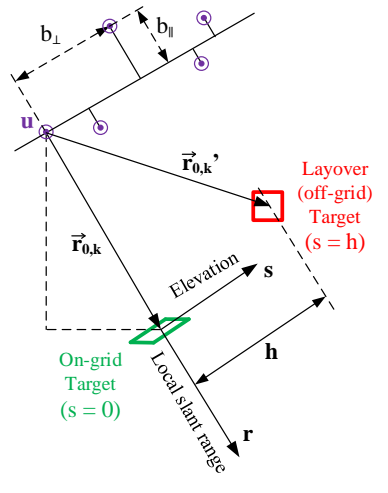

(b)
Fig. 2. (a) Refocusing geometry for a target with a known on-grid position $\overrightarrow{\boldsymbol{r}}_{\boldsymbol{k}}$. (b) Layover detection geometry based on zero elevation for on-grid targets.

(on-grid target situated at $\overrightarrow{\boldsymbol{r}}_{\boldsymbol{k}}$ ) the received signal in the $n$-th acquisition is written as:

$$
g_{n}\left(\overrightarrow{\boldsymbol{r}}_{\boldsymbol{k}}\right)=\int_{\delta s} \int_{\delta v} \gamma(s, v) \exp \left[j 2 \pi\left(\frac{2 b_{\perp, n}}{\lambda r_{0, k}} s+\frac{2 T_{n}}{\lambda} v\right)\right] d s d v
$$

where $\gamma(s, v)$ is the reflectivity function, $b_{\perp, n}$ is the orthogonal baseline computed for the target $k$ (perpendicular to $\overrightarrow{\boldsymbol{r}}_{\mathbf{0}, \boldsymbol{k}}$ ) relative to the first acquisition, $T_{n}$ is the acquisition time and $v$ is the mean displacement velocity (MDV). By changing the geometrical EV configuration for each refocused target, its position on the elevation axis is at $s=0$ and any other scatterer situated in layover will be at another distance as presented in Fig. 2(b). Therefore the coherent scatterers from the set of targets which have the real scattering center at the given position and are not affected by layover are the ones that have only one significant peak of the power spectral density (PSD) in the EV plane at zero elevation clearly separated from other eventual local peaks corresponding to layover targets.

\section{RESULTS AND DISCUSSION}

The proposed refocusing procedure was tested on a set of high-resolution spotlight images acquired with the TerraSARX satellite over the Puylaurent dam in France between MarchJune 2012. Due to the doppler centroid variation of spotlight images a few preprocessing steps described in [1] are necessary before defocusing. Also, in order to achieve an accuracy of the grid focusing in the centimeter level the distances computed in (15) and (16) have to be corrected with the annotated range and azimuth corrections (atmospheric shift and azimuth timing offset) [14]. The application of the refocusing procedure is illustrated in Fig. 3 for two different grid types: a rectangular grid and an irregular one. The rectangular grid is an uniformly spaced grid $(0.5 \mathrm{~m} \times 0.5 \mathrm{~m})$ aligned with the local latitude/longitude axis and situated at the height of the dam's ridge. The irregular grid consists of a set of differential GPS measured points on the water dam's ridge.

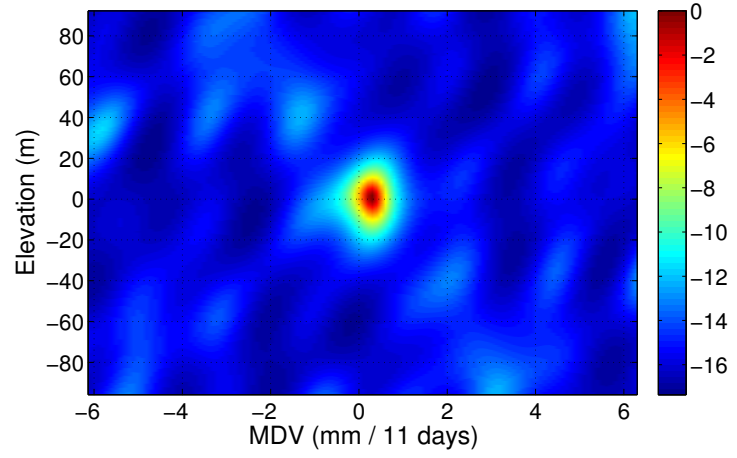

Fig. 4. Normalized PSD in the EV plane for a refocused point having one dominant scatterer.

TABLE I

Mean Displacement Velocity Measurement

\begin{tabular}{|c||c|c|}
\hline & $\begin{array}{c}\text { Estimated } \\
\text { (mm / 11 days) }\end{array}$ & $\begin{array}{c}\text { In-situ } \\
(\mathrm{mm} / 11 \text { days })\end{array}$ \\
\hline Central point & 0.60 & 0.68 \\
\hline Lateral point & 0.35 & 0.33 \\
\hline
\end{tabular}

Fig. 3(a) shows an image acquired by the TerraSAR-X satellite on the descending orbit on 11 March 2012. The refocused image on the rectangular grid is shown in Fig. 3(b) overlaid by the set of GPS measured points. Fig. 3(c) shows the same refocused image among with the detected scattering centers from the given set of points (the superimposed red points). For each refocused point an additional phase difference was applied using as reference a stable point situated at the dam's edge to compensate any residual propagation effects.

The PSD for each point was computed on a set of 8 images using adaptive beam-forming [5]. The covariance matrix was estimated for a given point among with 3 neighboring pixels from the refocused image on the rectangular grid. A typical normalized PSD obtained in the EV plane for one given point in the presence of only one dominant scatterer is shown in Fig. 4. Notice that the main lobe is situated around zero elevation and the MDV is not dispersed. Table I shows a comparison between the estimated and in-situ measured MDVs in line of sight (LOS) for two detected scattering centers situated in different regions of the dam where in-situ deformation data was available. An error below $0.1 \mathrm{~mm} / 11$ days (the satellite's revisit time) is plausible for a signal to noise ratio of around $15 \mathrm{~dB}$ (taking the power level in water as noise floor) and small data set (8 tracks).

\section{CONCLUSION}

A refocusing algorithm for SAR images on a given grid along with a differential tomography based scattering center detection was presented. The efficiency of the proposed method was tested on real data acquired with the TerraSAR$\mathrm{X}$ satellite. In future work, the time series for the detected scattering points will be determined starting from the presented framework. 


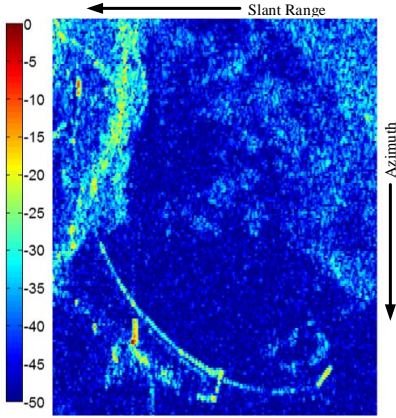

(a)

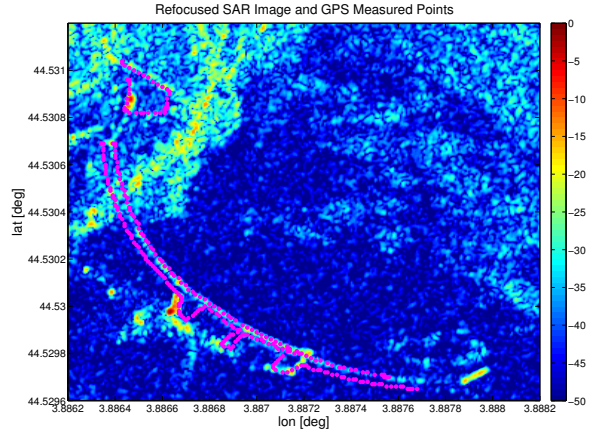

(b)

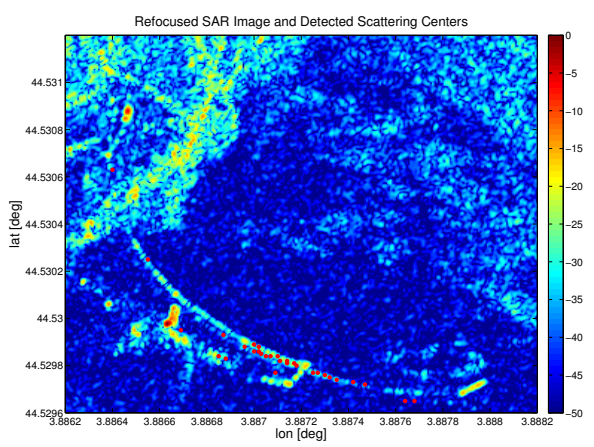

(c)

Fig. 3. The Puylaurent dam. (a) High-Resolution Spotlight TerraSAR-X SAR image acquired on 11 March 2012. (b) Refocused TerraSAR-X image on the rectangular grid overlaid by the set of GPS measured points on the ridge of the dam. (c) The refocused TerraSAR-X image on the rectangular grid among with the detected scattering centers from the refocused measured points on the dam's ridge (the superimposed red points).

\section{ACKNOWLEDGMENT}

The authors would like to thank the German Aerospace Center (DLR) for providing the TerraSAR-X and TanDEM-X SAR images through the MTH0828 and the NTIINSA2171 projects. Thanks to Dr. Irena Hajnsek (DLR) and Dr. Pau Prats (DLR) for the useful discussions regarding the refocusing of highresolution spotlight SAR images acquired in the TanDEM-X mission. The authors would also like to thank the Électricité de France (EDF) company for providing the in-situ measurements for the Puylaurent dam.

\section{REFERENCES}

[1] M. Eineder, N. Adam, R. Bamler, N. Yague-Martinez, and H. Breit, "Spaceborne spotlight sar interferometry with terrasar-x," Geoscience and Remote Sensing, IEEE Transactions on, vol. 47, no. 5, pp. 15241535, 2009.

[2] T. Fritz, C. Rossi, N. Yague-Martinez, F. Rodriguez-Gonzalez, M. Lachaise, and H. Breit, "Interferometric processing of tandem-x data," in Geoscience and Remote Sensing Symposium (IGARSS), 2011 IEEE International, 2011, pp. 2428-2431.

[3] F. Lombardini, "Differential tomography: a new framework for sar interferometry," Geoscience and Remote Sensing, IEEE Transactions on, vol. 43, no. 1, pp. 37-44, 2005.

[4] G. Fornaro, D. Reale, and F. Serafino, "Four-dimensional sar imaging for height estimation and monitoring of single and double scatterers," Geoscience and Remote Sensing, IEEE Transactions on, vol. 47, no. 1, pp. 224-237, 2009.

[5] F. Lombardini and M. Pardini, "Superresolution differential tomography: Experiments on identification of multiple scatterers in spaceborne sar data," Geoscience and Remote Sensing, IEEE Transactions on, vol. 50, no. 4, pp. 1117-1129, 2012.

[6] T. Fritz, H. Breit, and M. Eineder, "TerraSAR-X products tips and tricks," in TerraSAR-X Science Meeting 2008, DLR, Oberpfaffenhofen, Germany, 2008.

[7] S. Ugur, O. Arikan, and A. Gurbuz, "Off-grid sparse sar image reconstruction by emmp algorithm," in Radar Conference (RADAR), 2013 IEEE, 2013, pp. 1-4.
[8] A. Moreira, J. Mittermayer, and R. Scheiber, "Extended chirp scaling algorithm for air- and spaceborne sar data processing in stripmap and scansar imaging modes," Geoscience and Remote Sensing, IEEE Transactions on, vol. 34, no. 5, pp. 1123-1136, 1996.

[9] J. Mittermayer, A. Moreira, and O. Loffeld, "Spotlight sar data processing using the frequency scaling algorithm," Geoscience and Remote Sensing, IEEE Transactions on, vol. 37, no. 5, pp. 2198-2214, 1999.

[10] A. Moreira, "Real-time synthetic aperture radar (sar) processing with a new subaperture approach," Geoscience and Remote Sensing, IEEE Transactions on, vol. 30, no. 4, pp. 714-722, 1992.

[11] W. G. Carrara, R. S. Goodman, and R. M. Majewski, Spotlight Synthetic Aperture Radar: Signal Processing Algorithms. Boston: Artech House, 1995.

[12] M. Born and E. Wolf, Principles of Optics. Cambridge University Press, 1999, ch. Appendix 3.

[13] R. Raney, "A new and fundamental fourier transform pair," in Geoscience and Remote Sensing Symposium, 1992. IGARSS '92. International, vol. 1, 1992, pp. 106-107.

[14] T. Fritz, M. J., B. Schättler, B. W., S. Buckreuß, and R. Werninghaus, "TerraSAR-X ground segment level 1b product format specification," DLR, 2007. [Online]. Available: http://terrasar-x.dlr.de/

[15] P. Prats-Iraola, R. Scheiber, M. Rodriguez-Cassola, S. Wollstadt, J. Mittermayer, B. Brautigam, M. Schwerdt, A. Reigber, and A. Moreira, "High precision sar focusing of terrasar-x experimental staring spotlight data," in Geoscience and Remote Sensing Symposium (IGARSS), 2012 IEEE International, 2012, pp. 3576-3579.

[16] J. Munson, D.C., J. O'Brien, and W. Jenkins, "A tomographic formulation of spotlight-mode synthetic aperture radar," Proceedings of the IEEE, vol. 71, no. 8, pp. 917-925, Aug 1983.

[17] M. Desai and W. Jenkins, "Convolution backprojection image reconstruction for spotlight mode synthetic aperture radar," Image Processing, IEEE Transactions on, vol. 1, no. 4, pp. 505-517, Oct 1992.

[18] L. A. Gorham and L. J. Moore, "SAR image formation toolbox for matlab," vol. 7699, 2010, pp. 769 906-769906-13. [Online]. Available: http://dx.doi.org/10.1117/12.855375

[19] M. Albuquerque, P. Prats, and R. Scheiber, "Applications of timedomain back-projection sar processing in the airborne case," in Synthetic Aperture Radar (EUSAR), 2008 7th European Conference on, June 2008, pp. $1-4$. 